\title{
A Green's Function Method for the Vacuum Contribution to the MHD Stability of Helically Symmetric Equilibria
}

\author{
Peter Merkel \\ Max-Planck-Institut für Plasmaphysik EURATOM Association, Garching
}

Z. Naturforsch. 37 a, 859-866 (1982); received May 28, 1982

To Professor Arnulf Schlüter on his 60th Birthday

The HERA MHD stability code for helically symmetric equilibria has been supplemented by a code for computing the perturbation energy of a vacuum field surrounding the plasma and bounded by a conducting wall. By means of a Green's function technique the problem is reduced to the numerical solution of an integral equation. Extensive tests and convergence studies have been done to verify the code.

\section{Introduction}

The HERA stability code [1] computes the spectrum of the ideal MHD stability operator of helically symmetric equilibria. HERA is a modification of the ERATO code [2], which treats the stability of axisymmetric equilibria. In both codes two different procedures are provided for calculating the potential energy of a vacuum region bounded by the plasma surface and a surrounding conducting wall. The first approach treats the vacuum formally in the same way as the plasma by considering it as a shearless and pressureless plasma. The second method uses a Green's function technique. The problem is reduced to a coupled set of integral equations on the boundaries of the vacuum region. While the former approach has the advantage that vacuum and plasma region are treated in one step, the Green's function technique allows the case of a conducting wall at infinity to be easily solved.

In the present paper the application of the Green's function method to the helical case is described. In helical geometry the main obstacles are to find a sufficiently fast and accurate algorithm for computing the Fourier-transformed Green's function and its normal derivative and to treat properly the logarithmic singularities of the Green's function and its derivative. For the axisymmetric case in ERATO the singularities of the normal derivative are eliminated from the integral equations by subtracting known integrals with the same singularities [3], [4]. Here the Green's function and its

Reprint requests to Max-Planck-Institut für Plasmaphysik, Bibliothek, D-8046 Garching. normal derivative are split into a regular and a singular part. The numerical integration is then performed, using appropriate integration formulas for both types of integrands. This procedure works very well even when the wall comes close to the plasma surface.

\section{The Vacuum Potential Energy}

We consider a plasma configuration surrounded by a vacuum region bounded by an infinitely conducting shell (Figure 1). The vacuum field energy $\delta W_{\mathrm{v}}$ is given by

$$
\delta W_{\mathbf{v}}=\frac{1}{2} \int \mathrm{d}^{3} x(\delta \boldsymbol{B})^{2},
$$

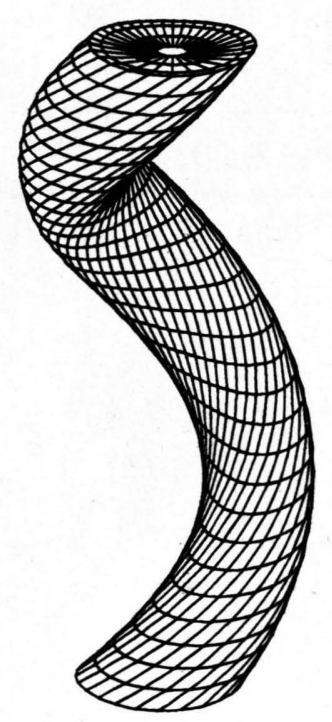

Fig. 1. The helical plasma configuration. 
where $\delta \boldsymbol{B}$, the perturbed magnetic vacuum field, satisfies

$$
\operatorname{div} \delta \boldsymbol{B}=0, \quad \operatorname{rot} \delta \boldsymbol{B}=0,
$$

and the boundary conditions

$$
\begin{aligned}
\boldsymbol{n} \cdot \delta \boldsymbol{B} & =0 \quad \text { on the shell } \\
\boldsymbol{n} \cdot \delta \boldsymbol{B} & =\operatorname{rot}\left(\boldsymbol{\xi} \times \boldsymbol{B}_{\mathrm{p}}\right) \cdot \boldsymbol{n} \\
& \text { on the plasma surface },
\end{aligned}
$$

where $\xi$ is the displacement vector of the perturbation and $\boldsymbol{B}_{\mathrm{p}}$ is the equilibrium magnetic field on the plasma surface [5], [6]. The normal unit vector $\boldsymbol{n}$ points towards the vacuum region.

The scalar potential $\Phi$ solves the Eqs. (2.2) with

$$
\delta \boldsymbol{B}=\boldsymbol{\nabla} \Phi
$$

and where $\Phi$ satisfies the Laplace equation

$$
\Delta \Phi=0 .
$$

The boundary conditions are

$$
\begin{aligned}
& \partial \Phi / \partial n=0 \quad \text { on the shell, } \\
& \partial \Phi / \partial n=\operatorname{rot}\left(\xi \times \boldsymbol{B}_{\mathrm{p}}\right) \cdot \boldsymbol{n}
\end{aligned}
$$

on the plasma surface.

Applying Green's theorem, the potential energy $\delta W_{\mathrm{v}}$ can be transformed into a surface integral over the plasma surface:

$$
\delta W_{\mathrm{v}}=\frac{1}{2} \int_{p} \Phi \frac{\partial \Phi}{\partial n} \mathrm{~d} f .
$$

To find $\delta W_{\mathrm{v}}$, one has to determine $\Phi$ on the plasma surface in terms of $\partial \Phi / \partial n$ which is given by (2.6).

This can be done by converting the differential equation for $\Phi(2.5)$ into an integral equation. It holds that

$$
\begin{aligned}
& \Phi(\boldsymbol{x})= \frac{1}{2 \pi} \\
& \cdot \int_{p}\left[\frac{\partial G\left(\boldsymbol{x}, \boldsymbol{x}^{\prime}\right)}{\partial n^{\prime}} \Phi\left(\boldsymbol{x}^{\prime}\right)-G\left(\boldsymbol{x}, \boldsymbol{x}^{\prime}\right) \frac{\partial \Phi\left(\boldsymbol{x}^{\prime}\right)}{\partial n^{\prime}}\right] \mathrm{d} f^{\prime} \\
& \\
&-\frac{1}{2 \pi} \int_{s} \frac{\partial G\left(\boldsymbol{x}, \boldsymbol{x}^{\prime}\right)}{\partial n^{\prime}} \Phi\left(\boldsymbol{x}^{\prime}\right) \mathrm{d} f^{\prime},
\end{aligned}
$$

with

$$
G\left(x, x^{\prime}\right)=1 /\left|x-x^{\prime}\right| .
$$

The $\boldsymbol{x}, \boldsymbol{x}^{\prime}$ are points on the boundary, i.e. on the plasma surface and the shell. Denoting the potential on the plasma surface as $\Phi_{\mathrm{p}}\left(\boldsymbol{x}_{\mathrm{p}}\right)$ and on the shell as $\Phi_{\mathrm{s}}\left(\boldsymbol{x}_{\mathrm{s}}\right)$, the integral equation divides into two coupled equations

$$
\begin{aligned}
\Phi_{\mathrm{p}}\left(\boldsymbol{x}_{\mathrm{p}}\right)= & \frac{1}{2 \pi} \int_{p}\left[\frac{\partial G\left(\boldsymbol{x}_{\mathrm{p}}, \boldsymbol{x}_{p}^{\prime}\right)}{\partial n^{\prime}} \Phi_{\mathrm{p}}\left(\boldsymbol{x}_{p}^{\prime}\right)\right. \\
& \left.-G\left(\boldsymbol{x}_{\mathrm{p}}, \boldsymbol{x}_{p}^{\prime}\right) \frac{\partial \Phi_{\mathrm{p}}\left(\boldsymbol{x}_{p}^{\prime}\right)}{\partial n^{\prime}}\right] \mathrm{d} f^{\prime} \\
& -\frac{1}{2 \pi} \int_{s} \frac{\partial G\left(\boldsymbol{x}_{\mathrm{p}}, \boldsymbol{x}_{s}^{\prime}\right)}{\partial n^{\prime}} \Phi_{\mathrm{s}}\left(\boldsymbol{x}_{s}^{\prime}\right) \mathrm{d} f^{\prime}
\end{aligned}
$$

and

$$
\begin{aligned}
\Phi_{\mathrm{S}}\left(\boldsymbol{x}_{\mathrm{s}}\right)= & \frac{1}{2 \pi} \int_{p}\left[\frac{\partial G\left(\boldsymbol{x}_{\mathrm{s}}, \boldsymbol{x}_{p}^{\prime}\right)}{\partial n^{\prime}} \Phi_{\mathrm{p}}\left(\boldsymbol{x}_{p}^{\prime}\right)\right. \\
& \left.-G\left(\boldsymbol{x}_{\mathrm{s}}, \boldsymbol{x}_{p}^{\prime}\right) \frac{\partial \Phi_{\mathrm{p}}\left(\boldsymbol{x}_{p}^{\prime}\right)}{\partial n^{\prime}}\right] \mathrm{d} f^{\prime} \\
& -\frac{1}{2 \pi} \int_{s} \frac{\partial G\left(\boldsymbol{x}_{\mathrm{s}}, \boldsymbol{x}_{s}^{\prime}\right)}{\partial n^{\prime}} \Phi_{\mathrm{s}}\left(\boldsymbol{x}_{s}^{\prime}\right) \mathrm{d} f^{\prime} .
\end{aligned}
$$

\section{Helical Coordinates and Fourier Transform}

Let us introduce helical coordinates $(r, \zeta, z)$, where the angular variable is $\zeta:=\varphi-h z$ and $(r, \varphi, z)$ are cylindrical coordinates. Helically symmetric equilibria are configurations which depend on $r$ and $\zeta$. The parameter $h$ controls the helical pitch.

The plasma surface and the conducting shell are defined by the closed curves in the plane $z=0$ :

$$
\begin{array}{ll}
r_{\mathrm{p}}=r_{\mathrm{p}}(\chi), & r_{\mathrm{s}}=r_{\mathrm{s}}(\chi), \\
\zeta_{\mathrm{p}}=\zeta_{\mathrm{p}}(\chi), & \zeta_{\mathrm{s}}=\zeta_{\mathrm{s}}(\chi),
\end{array}
$$

where $\chi$ is an appropriately chosen curve parameter.

The Green's function in helical coordinates is of the form

$$
\begin{aligned}
& G\left(r, \zeta, z ; r^{\prime}, \zeta^{\prime}, z^{\prime}\right) \\
& =\frac{1}{\left(r^{2}+r^{\prime 2}-2 r r^{\prime} \cos \left(\zeta-\zeta^{\prime}+h\left(z-z^{\prime}\right)\right)+\left(z-z^{\prime}\right)^{2}\right)^{1 / 2}} .
\end{aligned}
$$

To get the normal derivative $\partial G / \partial n$, one needs the operator $\mathrm{d} f \boldsymbol{n} \cdot \boldsymbol{\nabla}$ on the surfaces:

$$
\begin{aligned}
\mathrm{d} f \boldsymbol{n} \cdot \boldsymbol{\nabla}=\mathrm{d} \chi \mathrm{d} z(\dot{r} & r \frac{\partial}{\partial z}+r \dot{\zeta} \frac{\partial}{\partial r} \\
& \left.-\frac{\dot{r}}{r}\left(1+r^{2} h^{2}\right) \frac{\partial}{\partial \zeta}\right),
\end{aligned}
$$

where the dot is defined as $\cdot \equiv \partial / \partial \chi$. Applying this operator to $G$, one obtains $\partial G / \partial n \mathrm{~d} f$, where $\mathrm{d} f$ is the surface element. 
In the linearized stability analysis of helical equilibria all perturbed quantities are Fouriertransfcrmed with respect to the ignorable variable $z$. It is therefore sufficient to consider a vacuum potential of the form

$$
\Phi(\boldsymbol{x})=\Phi(r, \zeta) e^{i k z},
$$

where $k$ is the longitudinal wave number. Integrating (2.10) with respect to $z$, one obtains

$$
\begin{aligned}
\Phi_{\mathrm{p}}(\chi)= & \frac{1}{2 \pi} \int_{p}\left(G_{\mathrm{pp}}^{\mathrm{N}}\left(\chi, \chi^{\prime}\right) \Phi_{\mathrm{p}}\left(\chi^{\prime}\right)\right. \\
& \left.-G_{\mathrm{pp}}\left(\chi, \chi^{\prime}\right) \Phi_{\mathrm{p}}^{\mathrm{N}}\left(\chi^{\prime}\right)\right) \mathrm{d} \chi^{\prime} \\
- & \frac{1}{2 \pi} \int_{c} G_{\mathrm{ps}}^{\mathrm{N}}\left(\chi, \chi^{\prime}\right) \Phi_{\mathrm{s}}\left(\chi^{\prime}\right) \mathrm{d} \chi^{\prime}, \\
\Phi_{\mathrm{s}}(\chi)= & \frac{1}{2 \pi} \int_{p}\left(G_{\mathrm{sp}}^{\mathrm{N}}\left(\chi, \chi^{\prime}\right) \Phi_{\mathrm{p}}\left(\chi^{\prime}\right)\right. \\
& \left.-G_{\mathrm{sp}}\left(\chi, \chi^{\prime}\right) \Phi_{\mathrm{p}}^{\mathrm{N}}\left(\chi^{\prime}\right)\right) \mathrm{d} \chi \\
& -\frac{1}{2 \pi} \int_{s} G_{\mathrm{ss}}^{\mathrm{N}}\left(\chi, \chi^{\prime}\right) \Phi_{\mathrm{s}}\left(\chi^{\prime}\right) \mathrm{d} \chi^{\prime},
\end{aligned}
$$

where short notations are introduced for Green's function and potential to indicate that $G, G^{\mathrm{N}}$ and $\Phi$ are to be taken on the boundaries at points determined by $\chi, \chi^{\prime}$. One has

$$
\begin{aligned}
& G_{\mathrm{ab}}\left(\chi, \chi^{\prime}\right)=G_{k}\left(r_{\mathrm{a}}(\chi), \zeta_{\mathrm{a}}(\chi) ; r_{\mathrm{b}}^{\prime}\left(\chi^{\prime}\right), \zeta_{\mathrm{b}}^{\prime}\left(\chi^{\prime}\right)\right), \\
& G_{\mathrm{ab}}^{\mathrm{N}}\left(\chi, \chi^{\prime}\right)=G_{k}^{\mathrm{N}}\left(r_{\mathrm{a}}(\chi), \zeta_{\mathrm{a}}(\chi) ; r_{\mathrm{b}}^{\prime}\left(\chi^{\prime}\right), \zeta_{\mathrm{b}}^{\prime}\left(\chi^{\prime}\right)\right),
\end{aligned}
$$

where the Fourier-transformed Green's function and its normal derivative are defined by

$$
\begin{aligned}
& G_{k}\left(r, \zeta ; r^{\prime}, \zeta^{\prime}\right) \\
& =\int_{-\infty}^{+\infty} \mathrm{d} z \frac{e^{i k z}}{\left(r^{2}+r^{\prime 2}-2 r r^{\prime} \cos \left(\zeta-\zeta^{\prime}+h z\right)+z^{2}\right)^{1 / 2}}
\end{aligned}
$$

and

$$
\begin{aligned}
& G_{k}^{\mathrm{N}}\left(r, \zeta ; r^{\prime}, \zeta\right) \\
& =\left(-i k \dot{r}^{\prime} r^{\prime}+r^{\prime} \dot{\zeta}^{\prime} \frac{\partial}{\partial r^{\prime}}-\left(1+r^{\prime 2} h^{2}\right) \frac{\dot{r}^{\prime}}{r^{\prime}} \frac{\partial}{\partial \zeta^{\prime}}\right) G_{k} .
\end{aligned}
$$

The normal derivative of the potential $\Phi_{\mathrm{p}}^{\mathrm{N}}(\chi)$ in (3.5) is defined by

$$
\Phi_{\mathbf{p}}^{\mathrm{N}}(\chi) e^{i k z} \mathrm{~d} \chi \mathrm{d} z=\boldsymbol{n} \cdot \boldsymbol{\nabla}\left(\Phi_{\mathrm{p}}(r, \zeta) e^{i k z}\right) \mathrm{d} f .
$$

For the spectral energy density it follows from (2.7), (3.4) and (3.9) that

$$
\delta W_{k}=\pi \int_{p} \Phi_{p}^{\mathrm{N} *}(\chi) \Phi_{\mathrm{p}}(\chi) \mathrm{d} \chi .
$$

For HERA the vacuum energy has to be expressed in terms of the normal diplacement of the plasma $\boldsymbol{\xi} \cdot \boldsymbol{n}$ at the plasma surface, which is obtained by means of (2.6). It follows that

$$
\Phi_{\mathbf{p}}^{\mathrm{N}}(\chi) e^{i k z} \mathrm{~d} \chi \mathrm{d} z=\operatorname{rot}(\boldsymbol{\xi} \times \boldsymbol{B}) \cdot \boldsymbol{n} \mathrm{d} f,
$$

where the magnetic field is given by

$$
\begin{aligned}
\boldsymbol{B} & =T(\psi) \boldsymbol{u}+\boldsymbol{u} \times \nabla \psi, \\
\boldsymbol{u} & =\frac{1}{1+r^{2} h^{2}}\left(h r \boldsymbol{e}_{\varphi}+\boldsymbol{e}_{\mathrm{z}}\right) .
\end{aligned}
$$

Furthermore, flux coordinates are introduced: $\psi(r, \zeta), \chi(r, \zeta)$ with the Jacobian $J^{-1}=(\nabla \psi \times \nabla \chi) \boldsymbol{u}$. The poloidal variable $\chi$ is chosen such that $J u^{2}=$ $f(\psi)$ is a function of $\psi$ only [1]. On the plasma surface, the variable $\chi$ is identified with the previously defined curve parameter. With these definitions one obtains

$$
\Phi_{k}^{\mathrm{N}}(\chi)=\frac{1}{u^{2} J}\left(i k \tilde{q} X+\frac{\partial}{\partial \chi} X\right)
$$

with

$$
X=(\xi \nabla \psi) u^{2} J, \quad \tilde{q}=J u^{2}\left(T(\psi)-h r \frac{\partial \psi}{\partial r}\right) .
$$

\section{Properties of the Green's Function}

The numerical solution of the integral equations requires numerous evaluations of the Green's functions (proportional to $N^{2}$ ). A direct computation of the integrals (3.7) and (3.8) seems tedious and difficult. The integrands depend on $r, r^{\prime}, \zeta-\zeta^{\prime}$ and on the parameters $h$ and $k$, by which two different periodicities are introduced. Furthermore, the integrals converge very slowly as $\sin t / t$ for $t \rightarrow \infty$. Therefore, instead of computing these integrals, the Green's function and its normal derivative are transformed into a series of products of modified Bessel functions $I_{m}, K_{m}$.

By means of the expansion of the point charge Green's function in cylindrical coordinates [7]

$$
\begin{aligned}
\frac{1}{\left|\boldsymbol{x}-\boldsymbol{x}^{\prime}\right|}= & \frac{1}{\pi} \sum_{m=-\infty}^{+\infty} \int_{-\infty}^{+\infty} \mathrm{d} k \exp \left\{i m\left(\varphi-\varphi^{\prime}\right)\right\} \\
& \exp \left\{i k\left(z-z^{\prime}\right)\right\} b_{m}\left(|k| r,|k| r^{\prime}\right),
\end{aligned}
$$

where

$$
b_{m}\left(r, r^{\prime}\right)= \begin{cases}I_{m}(r) K_{m}\left(r^{\prime}\right), & r^{\prime} \geqq r, \\ I_{m}\left(r^{\prime}\right) K_{m}(r), & r^{\prime}<r,\end{cases}
$$


one finds for $G_{k}$ and $G_{k}^{\mathrm{N}}$ the Fourier series expansion

$$
\begin{aligned}
G_{k}\left(r, \zeta ; r^{\prime}, \zeta^{\prime}\right)= & 2 \sum_{m=-\infty}^{+\infty} \exp \left\{i m\left(\zeta-\zeta^{\prime}\right)\right\} \quad(4.3) \\
& \cdot b_{m}\left(r|k-m h|, r^{\prime}|k-m h|\right)
\end{aligned}
$$

and

$$
\begin{aligned}
& G_{k}^{\mathrm{N}}\left(r, \zeta ; r^{\prime}, \zeta^{\prime}\right)=2 \sum_{m=-\infty}^{+\infty} \exp \left\{i m\left(\zeta-\zeta^{\prime}\right)\right\} \\
& \cdot\left(-i h k \dot{r}^{\prime} r^{\prime}-i m\left(1+r^{\prime 2} h^{2}\right) \frac{\dot{r}^{\prime}}{r^{\prime}}+r^{\prime} \dot{\zeta} \frac{\partial}{\partial r^{\prime}}\right) \\
& \cdot b_{m}\left(r|k-m h|, r^{\prime}|k-m h|\right) .
\end{aligned}
$$

To study the convergence properties of these series and to find the singularities of these functions, the uniform asymptotic expansion of $b_{m}$ for $m \rightarrow \pm \infty$ is very useful. That expansion is found by means of the uniform asymptotic expansion of the modified Bessel functions, which are extensively discussed by Olver [8]; see also [9]. Taking these expansions, one gets for $b_{m}$ the following expression including all terms of the order $O\left(\mathrm{~m}^{-1}\right), O\left(\mathrm{~m}^{-2}\right)$ :

$$
\begin{aligned}
b_{\mathrm{m}}^{\text {asym }}= & \frac{1}{2|m|} \frac{1}{\left(\varrho \varrho^{\prime}\right)^{1 / 2}} \exp \left\{-|m|\left|\tau^{\prime}-\tau\right|\right\} \\
& \cdot \exp \left\{-\frac{m}{|m|} \frac{k}{h}\left|\varrho^{\prime}-\varrho\right|\right\} \\
& \cdot\left(1-\frac{1}{m} \frac{k}{2 h}\left(2-\frac{1}{\varrho^{\prime 2}}-\frac{1}{\varrho^{2}}\right)\right. \\
& -\frac{1}{|m|}\left(\frac{k^{2}}{2 h^{2}}\left|\frac{1}{\varrho^{\prime}}-\frac{1}{\varrho}\right|+\frac{1}{8} \mid \frac{1}{\varrho^{\prime}}\right. \\
& \left.\left.-\frac{1}{\varrho}\left|-\frac{5}{24}\right| \frac{1}{\varrho^{\prime 3}}-\frac{1}{\varrho^{3}} \mid\right)+\cdots\right)
\end{aligned}
$$

with

$$
\varrho=\left(1+r^{2} h^{2}\right)^{1 / 2}, \quad \tau=\varrho+\frac{1}{2} \ln \frac{\varrho-1}{\varrho+1},
$$

$\varrho^{\prime}, \tau^{\prime}$ are the same functions depending on $r^{\prime}$, $\varrho^{\prime}$ respectively.

The Green's functions $G_{k}$ and $G_{k}^{\mathrm{N}}$ become singular at $r=r^{\prime}, \zeta=\zeta^{\prime}$. These singularities are completely determined by the terms of the order $O\left(\mathrm{~m}^{-1}\right)$ and $O\left(m^{-2}\right)$ in $b_{m}$, because all other contributions converge for all values of the arguments. The singularities at $r=r^{\prime}, \zeta=\zeta^{\prime}$ do not depend on $k$; one can therefore set $k=0$. Taking for $b_{m}$ in (4.3) and (4.4) its asymptotic value $b_{\mathrm{m}}^{\text {asym }}$ and including all terms proportional to $m^{0}$ and $m^{-1}$ in $b_{\mathrm{m}}^{\text {asym }}$ and $\partial / \partial r^{\prime} b_{\mathrm{m}}^{\text {asym }}$, the series can be summed up in closed form.
The Fourier series (4.3) and (4.4) converge very fast for $r^{\prime} \mid r \ll 1$, as can be seen from the asymptotic expansion of $b_{m}$ (4.5). When $r^{\prime} \approx r$ and $\zeta \approx \zeta^{\prime}$, the convergence becomes very poor. To overcome this difficulty, two effective convergence accelerating procedures are provided. Firstly, series with the same asymptotic behaviour proportional to $m^{0}$, $m^{-1}, m^{-2}$ which can be summed up in closed form like (4.6) and (4.7) are subtracted. The remaining series then converges faster. Secondly, for the summation a convergence accelerating algorithm based on the theory of continued fraction [10] is applied.

The numerical solution of the integral equation requires computation of the Green's function for each pair of mesh cells, which gives a number proportional to $n_{\chi}{ }^{2}$. To reduce the number of Bessel function evaluations use was made of the factorial representation $I_{m}(r) K_{m}\left(r^{\prime}\right)$ in the terms of the sum. The modified Bessel functions are calculated in advance for all $2 n_{\chi}$ points for $m= \pm 1, \ldots, \pm 100$ and the results are stored. The only time consuming procedure then is the summation.

One obtains for the singular part of $G_{k}$ and $G_{\mathrm{R}}^{\mathrm{N}}$ :

$$
\begin{aligned}
& G^{\mathrm{sing}}\left(r, \zeta ; r^{\prime}, \zeta^{\prime}\right) \\
& =-2 \frac{\Re \mathrm{e} \ln \left(z^{\prime}-z\right)}{\left(1+r^{\prime 2} h^{2}\right)^{1 / 4}\left(1+r^{2} h^{2}\right)^{1 / 4}}
\end{aligned}
$$

and

$$
\begin{aligned}
& G^{\mathrm{N}, \operatorname{sing}}\left(r, \zeta ; r^{\prime}, \zeta^{\prime}\right) \\
& =-2 \Im \mathfrak{m} \frac{\dot{z}^{\prime}}{\left(z^{\prime}-z\right)}-\frac{1}{2} \dot{\zeta}^{\prime} \frac{r^{2} h^{2}}{\left(1+r^{2} h^{2}\right)} G^{\text {sing }}
\end{aligned}
$$

where $z$ is defined by

$$
\begin{aligned}
& z=\exp \{i \zeta-\tau\}, \\
& \tau=\sqrt{1+r^{2} h^{2}}+\frac{1}{2} \mathrm{n} \frac{h r}{1+\sqrt{1+r^{2} h^{2}}},
\end{aligned}
$$

and $z^{\prime}$ by the primed quantities.

\section{Discretization}

The boundaries are divided into $n_{\chi}$ non-aequidistant intervals (Figure 2). The intervals are defined by the nodal points $(0) j=1,2, \ldots$ and the physical points $(X)$ are given by the mid-points $j=1+1 / 2,2+1 / 2 \ldots$ The potential $\Phi$ and its normal derivative $\Phi^{\mathrm{N}}$ are expanded in terms of 


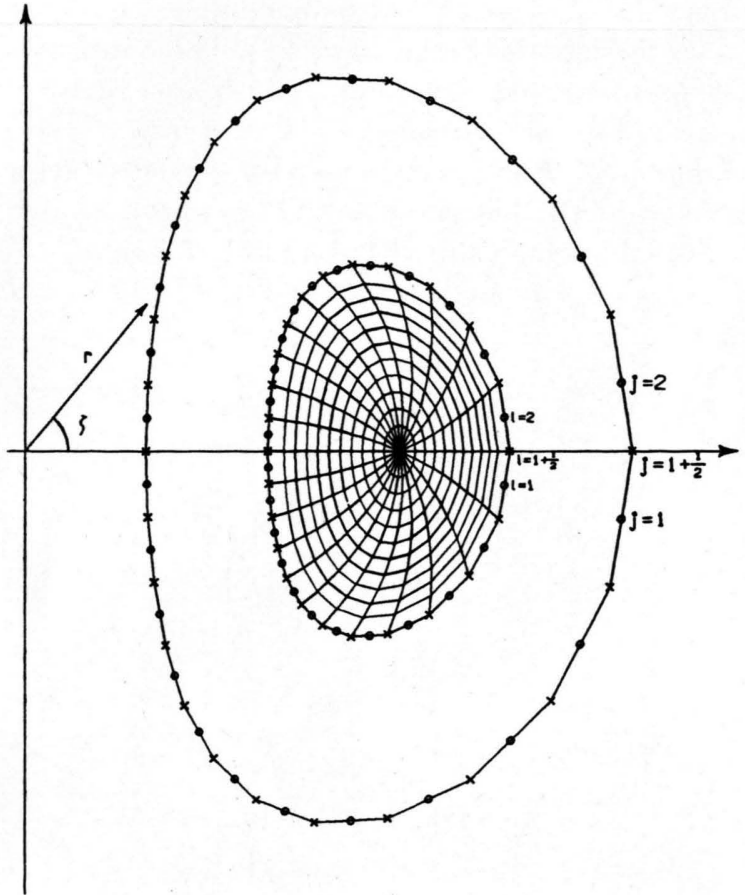

Fig. 2. The plasma cross-section, the surrounding conducting wall and the coordinate system.

piecewise constant finite elements $f_{j+1 / 2}$ :

$$
\begin{aligned}
& \Phi_{\mathrm{p}}(\chi)=\sum_{j=1}^{n_{\chi}} b_{j+1 / 2} f_{j+1 / 2}(\chi), \\
& \Phi_{\mathrm{s}}(\chi)=\sum_{j=1}^{n_{\chi}} c_{j+1 / 2} f_{j+1 / 2}(\chi), \\
& \Phi_{\mathrm{p}}^{\mathrm{N}}(\chi)=\sum_{j=1}^{n_{\chi}} a_{j+1 / 2} f_{j+1 / 2}(\chi),
\end{aligned}
$$

and

$$
f_{j+1 / 2}(\chi)= \begin{cases}1 & \text { for } \chi_{j}<\chi \leqq \chi_{j+1} \\ 0 & \text { otherwise }\end{cases}
$$

Inserting these expressions in the integral equations (3.5) and integrating over $\chi$, one obtains two coupled matrix equations:

$$
\begin{aligned}
& \boldsymbol{b}=\mathbf{A} \boldsymbol{b}-\mathbf{C} \boldsymbol{a}-\mathbf{B} \boldsymbol{c}, \\
& \boldsymbol{c}=\mathbf{D} \boldsymbol{b}-\mathbf{F} \boldsymbol{a}-\mathbf{E} \boldsymbol{c} .
\end{aligned}
$$

The complex matrix elements are given by

$$
\begin{aligned}
& A_{j l}=\frac{1}{2 \pi \Delta \chi_{j+1 / 2}} \int_{\chi_{j}}^{\chi_{j+1}} \mathrm{~d} \chi \int_{\chi_{l}}^{\chi_{l+1}} \mathrm{~d} \chi^{\prime} G_{\mathrm{pp}}^{\mathrm{N}}\left(\chi, \chi^{\prime}\right), \\
& B_{j l}=\frac{1}{2 \pi \Delta \chi_{j+1 / 2}} \int_{\chi_{j}}^{\chi_{j+1}} \mathrm{~d} \chi \int_{\chi_{l}}^{\chi_{l+1}} \mathrm{~d} \chi^{\prime} G_{\mathrm{ps}}^{\mathrm{N}}\left(\chi, \chi^{\prime}\right),
\end{aligned}
$$

$$
C_{j l}=\frac{1}{2 \pi \Delta \chi_{j+1 / 2}} \int_{\chi_{j}}^{\chi_{j+1}} \mathrm{~d} \chi \int_{\chi_{l}}^{\chi_{l+1}} \mathrm{~d} \chi^{\prime} G_{\mathrm{pp}}\left(\chi, \chi^{\prime}\right),
$$

where $\Delta \chi_{j+1 / 2}=\chi_{j+1}-\chi_{j}$.

The matrix elements of $\mathbf{D}, \mathbf{E}$ and $\mathbf{F}$ are obtained by replacing the first index $\mathrm{p}$ by $\mathrm{s}$ of the Green's functions in (5.4).

Eliminating $c$ from (5.3), the solution yields

$$
\begin{aligned}
\mathbf{b}= & \mathbf{Q} \boldsymbol{a}, \\
\mathbf{Q}= & \left((\mathbf{A}-\mathbf{I})-\mathbf{B}(\mathbf{E}+\mathbf{I})^{-1} \mathbf{D}\right)^{-1} \\
& \cdot\left(\mathbf{C}-\mathbf{B}(\mathbf{E}+\mathbf{I})^{-1} \mathbf{F}\right) a .
\end{aligned}
$$

where $\mathbf{I}$ is the unit matrix.

With (3.10), (5.1) and (5.5) the vacuum energy $\delta W_{k}$ is

$$
\delta W_{k}=\pi \sum_{j=1, l=1}^{n_{x}, n_{x}} a_{j+1 / 2}^{*} Q_{j l} a_{l+1 / 2} \Delta \chi_{j+1 / 2} .
$$

Finally, one has to express the $a_{j+1 / 2}$ by the normal displacement $X_{j}$ on the plasma surface. From (3.13) one derives the discretized equation

$$
\begin{aligned}
a_{j+1 / 2}= & i g_{j+1 / 2}^{(1)} \frac{X_{j+1}+X_{j}}{2} \\
& +g_{j+1 / 2}^{(2)} \frac{X_{j+1}-X_{j}}{\Delta \chi_{j+1 / 2}},
\end{aligned}
$$

with

$$
g_{j+1 / 2}^{(1)}=\left(\frac{k \tilde{q}}{J u^{2}}\right)_{j+1 / 2}, \quad g_{j+1 / 2}^{(1)}=\left(\frac{1}{J u^{2}}\right)_{j+1 / 2} .
$$

Defining a matrix $\mathbf{H}=\left\{\boldsymbol{H}_{j l}\right\}$ by

$$
\begin{aligned}
H_{j j} & =i \frac{1}{2} g_{j+1 / 2}^{(1)}-\frac{1}{\Delta \chi_{j+1 / 2}} g_{j+1 / 2}^{(2)}, \quad j=1, n_{\chi}, \\
H_{j j+1} & =-H_{j j}^{*}, \quad j=1, \quad n_{\chi}-1, \\
H_{n_{\chi 1}}: & =-H_{n_{x} n_{\chi}}^{*}, \quad H_{j l}=0 \quad \text { otherwise, }
\end{aligned}
$$

and a matrix $\hat{\boldsymbol{Q}}$ by

$$
\hat{Q}_{j l}=Q_{j l} \Delta \chi_{j+1 / 2},
$$

one ends up with

$$
\delta W_{k}=\boldsymbol{X}^{+} \mathbf{W} \boldsymbol{X}, \quad \text { with } \quad \mathbf{W}=\mathbf{H}^{+} \hat{\boldsymbol{Q}} \mathbf{H},(5.10)
$$

where $\boldsymbol{X}=\left\{X_{j}\right\}$ is the displacement vector. $\left(\mathbf{H}^{+}\right.$is the complex conjugate and transpose of $\mathbf{H}$.)

In the numerical program all $N$-dimensional complex vectors and $N \times N$ matrices are represented by $2 N$-dimensional real vectors and $2 N \times$ 
$2 N$ real matrices as follows:

$$
\boldsymbol{a}=\left\{\boldsymbol{a}^{\mathrm{R}}, \boldsymbol{a}^{\mathrm{J}}\right\}, \boldsymbol{Q}=\left(\begin{array}{rr}
\mathbf{Q}^{\mathrm{R}} & -\mathbf{Q}^{\mathrm{J}} \\
\mathbf{Q}^{\mathrm{J}} & \mathbf{Q}^{\mathrm{R}}
\end{array}\right),
$$

where $\boldsymbol{a}^{\mathrm{R}}\left(\boldsymbol{a}^{\mathrm{J}}\right)$ and $\mathbf{Q}^{\mathrm{R}}\left(\mathbf{Q}^{\mathrm{J}}\right)$ are the real (imaginary) parts of the vector and the matrix respectively.

\section{The Matrix Elements}

In order to integrate the matrix elements properly, the integrands are split into a regular part and a part, which can become singular:

$$
A_{j l}=A_{j l}^{\mathrm{reg}}+A_{j l}^{\mathrm{sing}} .
$$

The regular part obtained by subtracting the singular terms (4.6) and (4.7) from the Green's function (3.7) and its normal derivative (3.8) are integrated in lowest order using the midpoint rule. This approximation is sufficient because the potential $\Phi$ itself is approximated by a piecewise constant function resulting in quadratic convergence of the solution.

The regular matrix elements become

$$
\begin{aligned}
& A_{j l}^{\mathrm{reg}}=\frac{\Delta \chi_{l+1 / 2}^{\prime}}{2 \pi} \\
& \cdot\left(G_{\mathrm{pp}}^{\mathrm{N}}\left(\chi_{j+1 / 2}, \chi_{l+1 / 2}^{\prime}\right)-G_{\mathrm{pp}}^{\mathrm{N}, \operatorname{sing}}\left(\chi_{j+1 / 2}, \chi_{l+1 / 2}^{\prime}\right)\right) .
\end{aligned}
$$

To avoid numerical difficulties at the singular point $r=r^{\prime}, \zeta=\zeta^{\prime}$, the diagonal elements are treated in a different way when both variables belong to the same boundary:

$$
\begin{array}{r}
A_{j j}^{\mathrm{reg}}=\frac{\Delta \chi_{j+1 / 2}}{2 \pi} \frac{1}{4}((j, j+1)+(j, j-1) \\
+(6.3) \\
+(j+1, j)+(j-1, j)),
\end{array}
$$

where the notation used is

$$
\begin{aligned}
(j, l)= & G_{\mathrm{pp}}^{\mathrm{N}}\left(\chi_{j+1 / 2}, \chi_{l+1 / 2}^{\prime}\right) \\
& -G_{\mathrm{pp}}^{\mathrm{N}, \operatorname{sing}}\left(\chi_{j+1 / 2}, \chi_{l+1 / 2}^{\prime}\right) .
\end{aligned}
$$

The matrices $\mathbf{B}, \mathbf{C}, \mathbf{D}, \mathbf{E}, \mathbf{F}$ are treated analogously.

To obtain the singular part with the same accuracy, the logarithmic terms have to be properly integrated. The following integrals have to be taken:

and

$$
s\left(z_{+}^{\prime}, z_{-;}^{\prime} ; z_{+} z_{-}\right)=\int_{z_{-}^{\prime}}^{z_{+}^{\prime}} \mathrm{d} \chi^{\prime} \int_{z_{-}}^{z_{+}} \mathrm{d} \chi \ln \left(z^{\prime}-z\right)
$$

$$
g\left(z_{+}^{\prime}, z_{-}^{\prime} ; z_{+}, z_{-}\right)=\int_{z_{-}}^{z_{+}} \mathrm{d} \chi^{\prime} \int_{z_{-}^{\prime}}^{z_{+}} \mathrm{d} \chi \frac{\partial}{\partial \chi^{\prime}} \ln \left(z^{\prime}-z\right),
$$

where $z^{\prime}=z^{\prime}(\chi)$ and $z=z(\chi)$ are complex functions on the boundaries defined by (4.8). These integrals are approximated by formulas which are exact if $z^{\prime}$ and $z$ are linear functions of $\chi^{\prime}$ and $\chi$ respectively: $z=z_{0}+z_{1} \chi$. A regular function $f\left(z^{\prime}, z\right)$ as factor in front of the logarithm in (6.5) is approximated by its mid-point value. For $s$ and $g$ one gets

$$
\begin{aligned}
& s\left(z_{+}^{\prime}, z_{-}^{\prime} ; z_{+}, z_{-}\right)=\frac{1}{2} \Delta \chi \Delta \chi^{\prime} \\
& \cdot\left(-3-\frac{}{\left(z_{+}^{\prime}-z_{-}^{\prime}\right)\left(z_{+}-z_{-}\right)}\right. \\
& \quad \cdot\left(\left(z_{+}^{\prime}-z_{+}\right)^{2} \ln \left(z_{+}^{\prime}-z_{+}\right)+\left(z_{-}^{\prime}-z_{-}\right)^{2}\right. \\
& \quad \cdot \ln \left(z_{-}^{\prime}-z_{-}\right)-\left(z_{+}^{\prime}-z_{-}\right)^{2} \ln \left(z_{+}^{\prime}-z_{-}\right) \\
& \left.\left.\quad-\left(z_{-}^{\prime}-z_{+}\right)^{2} \ln \left(z_{-}^{\prime}-z_{+}\right)\right)\right)
\end{aligned}
$$

and

$$
\begin{aligned}
& g\left(z_{+}^{\prime}, z_{-}^{\prime} ; z_{+}, z_{-}\right)=\frac{\Delta \chi}{\left(z_{+}-z_{-}\right)} \\
& \cdot\left(\left(z_{+}^{\prime}-z_{-}\right) \ln \left(z_{+}^{\prime}-z_{-}\right)+\left(z_{-}^{\prime}-z_{+}\right)\right. \\
& \quad \cdot \ln \left(z_{-}^{\prime}-z_{+}\right)-\left(z_{+}^{\prime}-z_{+}\right) \ln \left(z_{+}^{\prime}-z_{+}\right) \\
& \left.\quad-\left(z_{-}^{\prime}-z_{-}\right) \ln \left(z_{-}^{\prime}-z_{-}\right)\right)
\end{aligned}
$$

where $\Delta \chi=\chi_{+}-\chi_{-}, \Delta \chi^{\prime}=\chi_{+}^{\prime}-\chi_{-}^{\prime}$.

The singular parts of the matrix elements are then $C_{j l}^{\text {sing }}$

$$
\begin{gathered}
=-\frac{\Re e s\left(z_{\mathrm{p} l+1}, z_{\mathrm{p} l} ; z_{\mathrm{p} j+1}, z_{\mathrm{p} j}\right)}{\pi \Delta \chi_{j+1 / 2}\left(1+r_{\mathrm{p} j+1 / 2}^{2} h^{2}\right)^{1 / 4}\left(1+r_{\mathrm{p} l+1 / 2}^{2} h^{2}\right)^{1 / 4}}, \\
A_{j l}^{\mathrm{sing}}=-\frac{1}{\pi \Delta \chi_{j+1 / 2}} \Im \mathrm{m} g\left(z_{\mathrm{p} l+1}, z_{\mathrm{p} l} ; z_{\mathrm{p} j+1}, z_{\mathrm{p} j}\right), \\
\quad-\frac{r^{2}{ }_{p j+1 / 2} h^{2}}{2\left(1+r_{p j+1 / 2}^{2}\right) h^{2}} \dot{\zeta}_{\mathrm{p} l+1 / 2} C_{\mathrm{jl}}^{\mathrm{sing}}, \\
B_{j l}^{\operatorname{sing}} \\
=\frac{\Re \operatorname{le} s\left(z_{\mathrm{p} l+1}, z_{\mathrm{p} l} ; z_{\mathrm{s} j+1}, z_{\mathrm{s} j}\right)}{\pi \Delta \chi_{j+1 / 2}\left(1+r^{2}{ }_{\mathrm{s} j+1} h^{2}\right)^{1 / 4}\left(1+r^{2}{ }_{\mathrm{p} l+1 / 2} h^{2}\right)^{1 / 4}} .
\end{gathered}
$$

The diagonal elements of the matrices for which both variables belong to the same boundary have to be considered by particular care. Because the principal values of the integrals have to be taken, one gets

$$
C_{j j}^{\text {sing }}=\frac{\Delta \chi_{j+1 / 2}}{2 \pi} \frac{\Re e\left(-3+2 \ln \left(z_{\mathrm{p} j+1}-z_{\mathrm{p} j}\right)\right)}{\left(1+r^{2}{ }_{\mathrm{p} j+1} h^{2}\right)^{1 / 2}},
$$

$$
A_{j j}^{\mathrm{sing}}=\frac{r_{\mathrm{p} l+1 / 2}^{2} h^{2}}{2\left(1+r_{\mathrm{p} j+1 / 2}^{2} h^{2}\right)} \dot{\zeta}_{\mathrm{p} j+1 / 2} C_{j j}^{\mathrm{sing}} .
$$


The singular parts of $\mathbf{D}, \mathbf{E}$ and $\mathbf{F}$ are obtained by replacing the index $\mathrm{p}$ by $\mathrm{s}$ in all $j$ terms.

\section{Test Results}

The procedure presented for computing the vacuum field perturbation energy is installed in the HERA code and has been successfully applied to stability studies of helical equilibria [10]. A typical cross-section and a view of the plasma surface of such an equilibrium are shown in Figs. 1 and 2. These equilibria are surrounded by a vacuum bounded by a conducting wall of the same shape as the plasma surface, similarly enlarged by a factor $R_{\text {ext }}$.

In the continuum limit the energy matrix $\mathbf{W}$ in (5.10) has to be Hermitian, which is only approximately satisfied in a finite mesh. A measure of the deviation from that symmetry is given by

$$
\sigma^{2}=\frac{\sum_{j, l=1}^{n_{x}}\left(W_{j l}-W_{l j}^{*}\right)\left(W_{j l}-W_{l j}^{*}\right)}{\sum_{j, l=1}^{n_{x}} W_{j l} W_{j l}^{*}} .
$$

In Fig. 3 the symmetry function $\sigma^{2}$ is plotted as a function of $n_{\chi}^{-4}$ for a case with $R_{\text {ext }}=2$.

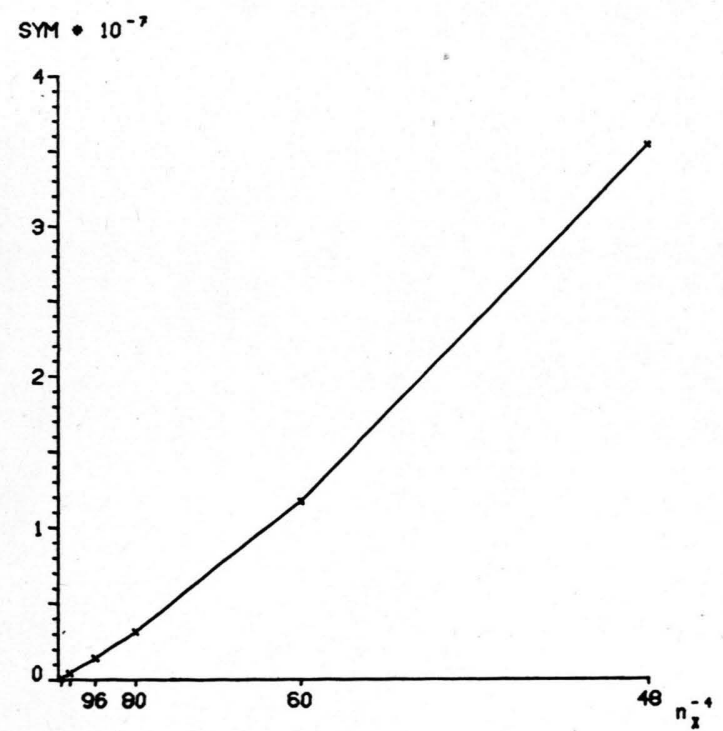

Fig. 3. The asymmetry $\sigma^{2}(=\mathrm{SYM})$ of the $W$ matrix as function of the number of intervals $n_{\chi}$.

[1] R. Gruber, S. Semenzato, F. Troyon, T. Tsunematsu, W. Kerner, P. Merkel, and W. Schneider, Comput. Phys. Commun. 24, 363 (1981).

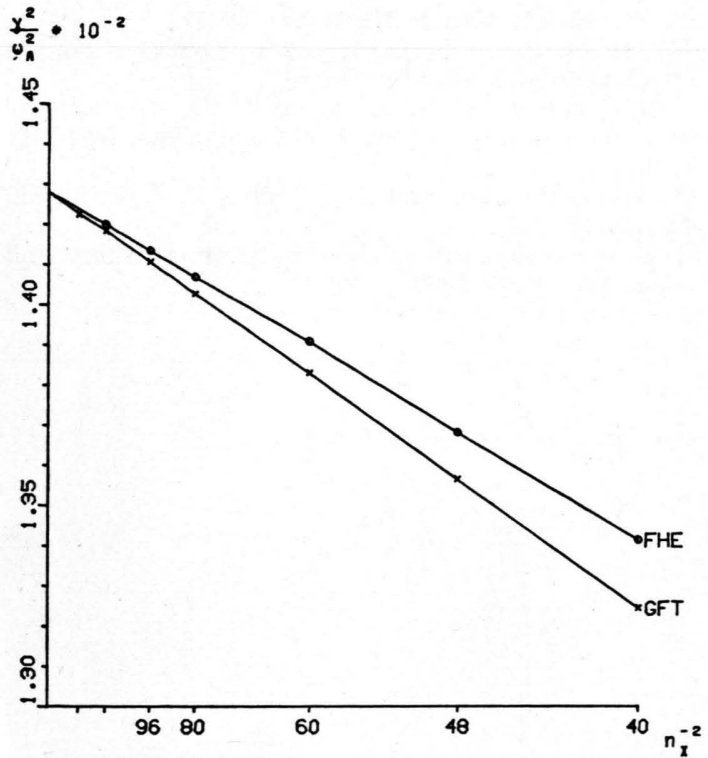

Fig. 4. The eigenvalues $\gamma^{2}$ of an $m=1, k=0.92$ mode for an equilibrium with $\langle\beta\rangle=0.21, R_{\text {ext }}=2$, are plotted as function of $n_{\chi}^{-2}$ (see [11]). Comparison between the results obtained with the Green's function technique (GFF) and the plasma-like treatment (GHE) of the vacuum [1].

The error with respect to the symmetry is of the order $O\left(n_{\chi}^{-4}\right)$, a result which has been found in the axisymmetry case as well [4]. The HERA code requires a symmetric matrix $\mathbf{W}$. Therefore, $\mathbf{W}$ is replaced by the symmetrized matrix $\bar{W}=1 / 2$ $\left(\mathbf{W}+\mathbf{W}^{+}\right)$, which is justified by the small symmetry error.

In Fig. 4 the eigenvalues $\gamma^{2}$ of an $m=1$ mode $(2 m$ - poloidal node number) are plotted versus $n_{\chi}^{-2}$. The results show the expected quadratic convergence. Given for comparison are the corresponding values obtained by the alternative vacuum program, which treats the vacuum as a pseudoplasma [1]. Both curves converge to the same eigenvalue.

\section{Acknowledgements}

The author gratefully acknowledges fruitful discussions with R. Gruber, J. Nührenberg and F. Troyon and would like to thank R. Großmann for carrying out part of the numerical calculations.

[2] R. Gruber, F. Troyon, D. Berger, L. C. Bernard, S. Rousset, R. Schreiber, W. Kerner, W. Schneider, and K. V. Roberts, Comput. Phys. Commun. 21, 323 (1981). 